\title{
Challenges for Enhanced Network Self-Manageability in the Scope of Future Internet Development
}

\author{
Ioannis P. Chochliouros ${ }^{1, *}$, Anastasia S. Spiliopoulou $^{2}$, and Nancy Alonistioti ${ }^{3}$ \\ ${ }^{1}$ Head of Research Programs Section, Network Strategy and Architecture Dept., \\ Hellenic Telecommunications Organization S.A. (OTE), \\ 99 Kifissias Avenue, 15124 Maroussi, Athens, Greece \\ ichochliouros@oteresearch.gr \\ ${ }^{2}$ Lawyer, General Directorate for Regulatory Affairs, \\ Hellenic Telecommunications Organization S.A. (OTE), \\ 99 Kifissias Avenue, 15124 Maroussi, Athens, Greece \\ aspiliopoul@ote.gr \\ ${ }^{3}$ Lecturer, National and Kapodistrian University of Athens, \\ Dept. of Informatics and Communications, 15784, Panepistimiopolis, Ilissia, Athens, Greece \\ nancy@di.uoa.gr
}

\begin{abstract}
The work examines perspectives from the inclusion of the autonomicity and self-manageability features in the scope of Future Internet's (FI) deployment. Apart from the strategic importance for further evolution, we also discuss some major future challenges among which is the option for an effective network management (NM), as FI should possess a considerably enhanced network manageability capability. We examine a new network manageability paradigm that allows network elements (NEs) to: be autonomously interrelated/controlled; be dynamically adapted to changing environments, and; learn the desired behaviour over time, based on the original context of the Self-NET research project effort. As self-organizing and self-managing systems have a considerable market impact, we identify benefits for all market actors involved. In addition, we incorporate some recent, but very promising experimental findings, mainly based on the context of a specific use-case for network coverage and capacity optimization, highlighting the way towards developing specific NM-related solutions, able to be adopted by the real market sector. We conclude with some essential arising issues.
\end{abstract}

Keywords: Autonomicity, cognitive networks, Future Internet (FI), network manageability, Network Management (NM), self-configuration, self-manageability, self-management, situation awareness (SA).

\section{Introduction - Moving Towards the Future Internet}

There is an extensive consensus that the Internet, as one of the most critical infrastructures of the $21^{\text {st }}$ century, can critically affect traditional regulatory theories as

* Corresponding Author. 
well as existing governance practices [1]. But, as the future of the Internet comes into consideration, in parallel with the appearance and/or the development of modern infrastructures, even greater challenges appear, with many concerns relevant to privacy, security and governance and with a diversity of issues related to Internet's effectiveness and inclusive character. Future related facilities will "attract" more users to innovative services requiring greater mobility and bandwidth, higher speeds and improved interactivity through the launch of many interactive media- and contentbased applications [2]. Nevertheless, such claims necessitate a more secure, reliable, scalable and easily manageable Internet architecture. If well deployed, the Internet of the future can bring novelty, productivity gains, new markets and growth.

In fact, innovative functionalities with more enhanced performance levels are necessary to sustain the real-time requirements of a multitude of novel applications. Furthermore, the Internet underpins the whole global economy. The diversity and sheer number of applications and business models supported by the Internet have also largely affected its nature and structure ([3], [4]).

The Future Internet (FI) will not be "more of the same", but rather "appropriate entities" incorporating new technologies on a large scale that can unleash novel classes of applications and related business models [5]. If today's Internet is a crucial element of our economy, FI will play an even more vital role in every conceivable business process. It will become the productivity tool "par excellence". At present, there are many so called "Future Internet" initiatives around the world working on defining and implementing a new architecture for the Internet intended to overcome existing limitations mostly in the area of networking ([6], [7]). The complexity of the FI, bringing together large communities of stakeholders and expertise, requires a structured mechanism to avoid fragmentation of efforts and to identify goals of common interest. Appropriate action is therefore invaluable to pull together the different initiatives, in order to provide more potential options and/or opportunities for the market players involved. Europe remains an international force in advanced information and communication technologies (ICT) and has massively adopted broadband and Internet services [8]. The European Union (EU) is actually a potential leader in the FI sector [9]. Leveraging FI technologies through their use in "smart infrastructures" offer the opportunity to boost European competitiveness in emerging technologies and systems, and will make it possible to measure, monitor and process huge volumes of information. This can also give the means to "overcome" fragmentation and to construct a related critical mass at European level, while fostering competition, openness and standardisation, involving consumer/citizen, ensuring trust, security and data protection with transparent and democratic governance and control of offered services as guiding principles ([10], [11]).

\subsection{Autonomicity and Self-Management Features in Modern Network Design}

The face of the Internet is continually changing, as new services appear and become globally noteworthy, while market actors are adapting to these challenges through suitable business models [12]. The current Internet has been founded on a basic architectural premise, that is: a simple network service can be used as a "universal means" 
to interconnect intelligent end systems [13]. Thus, it is centred on the network layer being capable of dynamically selecting a path from the originating source of a packet to its ultimate destination, with no guarantees of packet delivery or traffic characteristics. The continuation of simplicity in the network has pushed complexity into the end-points, thus allowing Internet to reach an impressive scale in terms of interconnected devices. However, while the scale has not yet reached its limits, the growth of functionality and the growth of size have both slowed down. It is now a common belief that current Internet is reaching both its architectural capability and its capacity limits (i.e.: addressing, reachability, new demands on quality of service (QoS), service/application provisioning, etc.). The next generation network architecture will be flexible enough to support a range of application visions in a dynamic way, ensuring convergence between technology, business and regulatory concerns. Enhanced communication services will open many possibilities for innovative applications that are not even envisioned today. Challenges for the Network of the Future may refer to a great variety of factors, including but not limited to: Dependability and security; scalability; services (i.e.: cost, service-driven configuration, simplified services composition over heterogeneous networks, large scale and dynamic multi-service coexistence, exposable service offerings/catalogues); monitoring; Service Level Agreements (SLAs) and protocol support for bandwidth (dynamic resource allocation), latency and QoS; automation (e.g. automated negotiation), and; the option for autonomicity. The resolution of these challenges would bring benefits to network and to serviceapplication providers, in terms of: Simplified contracting of new business; establishing/identifying reference points for resource allocation and re-allocation; enabling flexibility in the provisioning and utilization of resources; offering the ability to scale horizontally, and; providing a natural complement to the virtualization of resources by setting up and tearing down composed services, based on negotiated SLAs. This also involves benefits for service providers/consumers, in terms of: Ready identification-selection of offerings; potential to automate the negotiation of SLA Key Performance Indicators (KPIs) and pricing; reduced cost and time-to-market for services; scalability of composed services, and; flexibility and independence from the underlying network details.

In addition, a current trend for networks is that they are becoming service-aware. Service awareness itself has many aspects, including the delivery of content and service logic, fulfilment of business and other service characteristics such as QoS and SLAs and the optimization of the network resources during the service delivery. Thus, the design of networks and services is moving forward to include higher levels of automation, autonomicity, including self-management. Conversely, services themselves are becoming network-aware. Networking-awareness means that services are executed and managed within network execution environments and that both services and network resources can be managed uniformly in an integrated way. It is commonly acknowledged that the FI should have a considerably enhanced network manageability capability, and be an inseparable part of the network itself. Manageability of the current network typically resides in client stations and servers, which interact with network elements (NEs) via protocols such as SNMP (Simple Network Management Protocol). The limitations of this approach are reduced scaling properties to 
large networks and the need for extensive human supervision and intervention. A new network manageability paradigm is thus needed that allows NEs to be autonomously interrelated and controlled; adapts dynamically to changing environments, and; learns the desired behaviour over time. The effective design of monitoring protocols so as to support detection mechanisms critical for the elaboration of self-organizing networks has to be based on a clear understanding of engineering "trade-offs" with respect to local vs. non-local and aggregated information, for instance. In fact, several issues identified in current network infrastructures impose the need for the introduction of an innovative architectural design. Furthermore, the diversity of services as well as the underlying hardware and software resources comprise management issues highly challenging, meaning that currently, a diversity in terms of hardware resources leads to a diversity of management tools (distinguished per vendor). In addition, security risks currently present in network environments request for immediate attention. This could be achieved by building trustworthy network environments to assure security levels and manage threats in interoperable frameworks for autonomous monitoring.

\subsection{The Vision of a Modern Self-Managing Network}

The future vision is that of a self-managing network whose nodes/devices are designed in such a way that all the so-called traditional network management functions, defined by the "FCAPS" management framework (Fault, Configuration, Accounting, Performance and Security) [14], as well as the fundamental network functions such as routing, forwarding, monitoring, discovery, fault-detection and fault-removal, are made to automatically "feed" each other with information such as goals and events, to effect feedback processes among the different functions. Such processes allow reactions of various functions in the network (also including its individual nodes/devices), to achieve and maintain well-defined network goals [15].

Self-management capabilities may relate to a great variety of significant issues, such as: (i) Cross-domain management functions, for networks, services, content, together with the design of cooperative systems providing integrated management functionality of system lifecycle, self-functionality, SLA and QoS; (ii) Embedded management functionality in all FI systems (such as: in-infrastructure/in-network/inservice and in-content management); (iii) Mechanisms for dynamic deployment of new management functionality without interruption of actually running systems; (iv) Mechanisms for dynamic deployment of measuring and monitoring probes for services'/network's behaviour, including traffic; (v) Mechanisms for conflict and integrity-issues detection/resolution across multiple self-management functions; (vi) Mechanisms, tools and methodology construction for the verification and assurance of diverse self-capabilities that are "guiding systems" and their adaptations, correctly; these can also relate to mechanisms for allocation \& negotiation of different available resources; (vii) Increased level of self -awareness/-knowledge/-assessment and selfmanagement capabilities for FI resources; (viii) Increased level of self-adaptation and self-composition of resources to achieve autonomic and controllable behaviour; (ix) Increased level of resource management, including discovery, deployment utilization, configuration, control and maintenance; (x) Self-awareness capabilities to support 
objectives of minimizing system life-cycle costs and energy footprints; (xi) Orchestration and/or integration of management functions, and; (xii) Capabilities for the control relationships between self-management and self -governance of the FI.

In such an evolving environment, it is required the network itself to help detect, diagnose and repair failures, as well as to constantly adapt its configuration and optimize its performance. Looking at Autonomicity and Self-Manageability, the former (i.e. control-loops and feed-back mechanisms/processes, as well as the information/knowledge flow used to drive control-loops), becomes an enabler for network self-manageability [16]. Furthermore, new wireless sensor network technologies provide options for inclusion of additional intelligence and the capability, for the network elements and/or domains to "sense, reason and actuate". Suitable systems with communication and computational capabilities can be integrated into the fabric of the Internet, providing an accurate reflection of the real world, delivering fine-grained information and enabling almost real-time interaction between the virtual world and real world. In particular, autonomous self-organizing systems are beginning to emerge and to be widely established [17]. Such systems "can adapt autonomously" to changing requirements and reduce the reliance on centrally planned services, especially if they are effectively joined with new network management techniques. Operators may use these tools to guarantee QoS service in a period of exploding demand and rising network congestion at peak times. The trend in building dependable real-life systems and smart infrastructures today is "to move from monolithic, centralized and strictly hierarchical systems to highly distributed networked systems with local and global autonomy". When they are deployed in complex processes, these systems exhibit promising features and capabilities such as modularity and scalability, low cost, robustness and adaptability. Some of the challenges for operators/service providers include management (especially in self-organized wireless environments), resilience and robustness, automated re-allocation of resources, operations' abstractions in the underlying infrastructure, QoS guarantees for bundled services and optimization of operational expenditures (OPEX).

Ubiquitous and self-organizing systems are not only disruptive technologies that impact the way how market actors organize core processes as well as existing structures in value chains and industry, but have also considerable impact. The present Internet model is based on clear separation of concerns between protocol layers, with intelligence moved to the edges, and with the existent protocol pool targeting user and control plane operations with less emphasis on management tasks [18]. The area of FI is considered as a representative example of a "complex adaptive organization" (or "entity"), where the involved partners have diverse goals and tension to maximize their gains. There is a need for new ways to organize, control and structure communication systems, according to new management schemes and networking techniques without neglecting the advantages of current Internet. Among the core drivers for the FI are increased reliability, enhanced services, more flexibility, and simplified operation. The latter calls for including Network Management (NM) issues into the design process for FI principles. In general, NM is a service (or application) that employs a diversity of tools, applications, and devices to assist human network managers in monitoring and maintaining networks. Thus, NM should be an integral part of the 
future network infrastructure. Management is a key factor in manageability, usability, performance, etc., and is an important factor to the operational costs of any "network entity". FI requires a new management approach, promoted mainly by the necessity of support interoperability between heterogeneous, complex and distributed systems, while it should remain open for further and continuous improvement without the necessity of another disruptive modification in the future. Furthermore, as NM is important for the reliable and safe operation of networks, it is also crucial for the success of the FI. In the scope of these challenges, the Self-NET Project (https://www.ict-selfnet.eu/) aims to integrate the self-management and cognition features and the inevitable part of FI evolution.

\section{Network Management Activities in the Self-NET Scope}

The Self-NET Project designs, develops and validates an innovative paradigm for cognitive self-managed elements of the FI. Self-NET engineers the FI, based on cognitive behaviour with a high degree of autonomy [19] by proposing and examining the operation of self-managed FI elements around a novel "feedback-control cycle" (i.e. the "Monitoring/Decision-Making/Execution" or "MDE" cycle) as shown in Fig.1. Thus, dynamic distribution of resources according to network needs at specific time intervals can be pursued by introducing the "MDE" cycle to overcome bottlenecks and ensure seamless service provisioning - even in case of services with high bandwidth requirements. The completion of the aforementioned objective can make certain better QoS, beyond the original best-effort status, and simultaneously eases operational and network management functionalities.

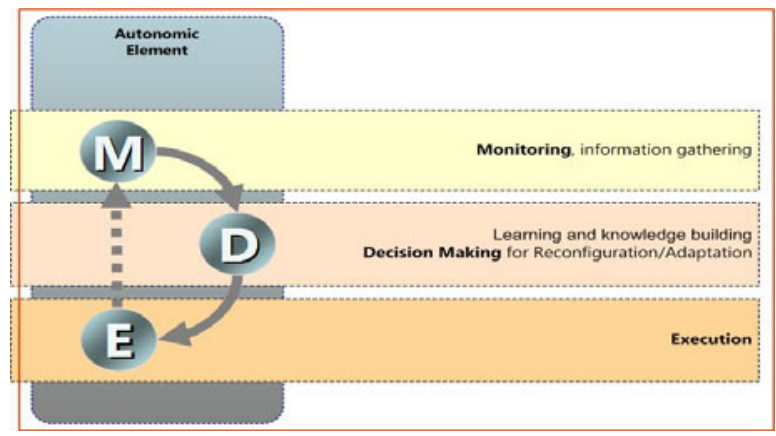

Fig. 1. The Distributed Cognitive Cycle for Systems and Network Management (DC-SNM)

Cognitive management in FI elements introduces innovative techniques regarding converged infrastructures with ultra-high capacity access networks and converged service capability across heterogeneous environments. Besides, the introduction of cognition in networks can contribute towards overcoming structural limitations of current infrastructures -which render it difficult to cope with a wide variety of networked applications, business models, edge devices and infrastructures- so as to guarantee higher levels of scalability, mobility, flexibility, security, reliability and 
robustness. Self-NET principle design is based on high autonomy of NEs in order to allow distributed management, fast decisions, and continuous local optimization of existing networks or of specific network parts [20]. The three distinct phases of the Generic Cognitive Cycle Model-GCCM (i.e.: the MDE cycle) are as shown in Fig. 2.

Cognitive capabilities can enable the perception of the NEs environment and the decision upon the necessary action (e.g. configuration, healing, protection measures, etc.). As current management tasks are becoming overwhelming, Self-NET embeds new management capabilities into NEs to take advantage of the increasing knowledge that characterizes the daily operation of FI users [21]. Among the main Self-NET's efforts is "to tackle complexity" by following the well-known "divide and conquer" approach, that is by: "Breaking down the overall network management task into smaller manageable tasks" and assigning them to individual NEs; showing NEs how to tackle the relevant issues; giving NEs the ability to "learn" in order to solve new, emerging (and occasionally "unforeseen") problems; facilitating NEs to cooperatively solve problems that require a sort of coordination, and; enhancing FI with inherent management capabilities (i.e. "making FI self-manageable"). NEs with cognitive capabilities aim at fast localised decision-making and (re-)configuration actions, as well as learning capabilities that improve elements behaviour. An essential target of the Project effort is to develop innovative cross-layer design optimization approaches that alleviate the shortcomings and duplication of functionalities in different protocol layers of the present IP stack. Furthermore, Self-NET also provides a peer-to-peer style distribution of responsibilities among self-governed FI elements, therefore overcoming the barrier of current client-server and proxy-based models in the operation of mobility management, broadcast-multicast, and QoS mechanisms. A "key-objective" is the provision of a holistic architectural \& validation framework that unifies networking operations and service facilities [22]. FI design is required to provide answers to a number of current Internet's deficits, especially when the danger of increased complexity is more than evident. Self-management and autonomic capabilities can so alleviate this "drawback" by: providing inherent management capabilities; increasing flexibility, and; allowing an ever-evolving Internet. Towards realizing this aim, Self-NET considers that a DC-SNM along with a hierarchical distribution over the network can "map" self-management capabilities over FI architectures [23]. DCSNM further facilitates the promotion of distributed-decentralized management over a hierarchical distribution of management and (re-) configuration making levels: (i) to (autonomic) NEs; (ii) to network domain types, and; (iii) up to the service provider realm, hence allowing high autonomy of NEs with cognitive capabilities aimed at fast localised (re-)configuration actions and decision-making. This brings about the intriguing issue of orchestrating the cognitive cycles (MDE) at higher levels of the selfmanagement distribution.

The "decomposition" of NM into responsibility areas (as shown in Fig.2) can provide the principle on which universal management architecture can be developed, having as a main goal the efficient handling of complexity towards FI environments. This, combined with the introduction of cognitive functionalities at all layers, can allow decisions/configurations at shorter time-scales [24], where each element has embedded cognitive cycle functionalities and also the ability to manage itself and 
make appropriate local decisions. For an efficient and scalable NM, where various actors may participate, a distributed approach is thus adopted. Dynamic network (re-) configuration in many cases is based on cooperative decision of various FI elements and distributed NM service components. Hints and requests/recommendations are exchanged among the related layers, in order to "identify" a new situation-action for a "targeted" execution. The automated (dynamic) incorporation of various layers requirements into the management aspects also provides novel features to NM [25].

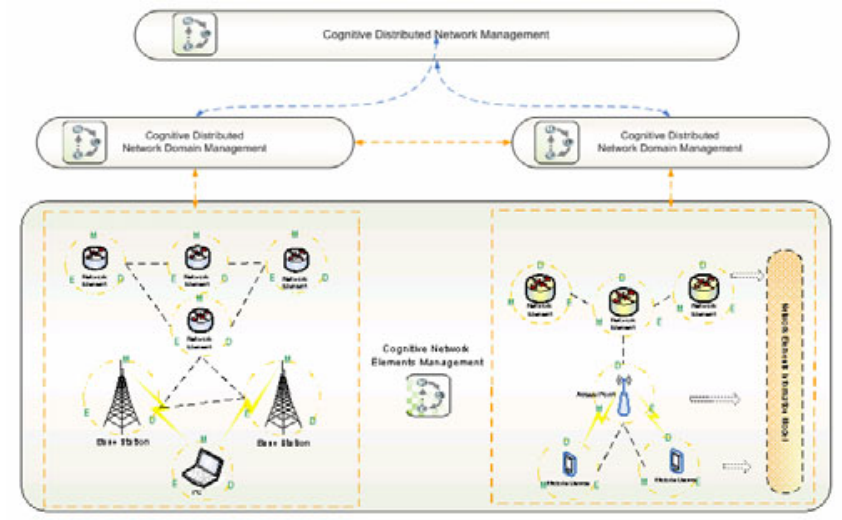

Fig. 2. The Distributed Cognitive Cycle for Systems and Network Management (DC-SNM).

In the context of the Self-NET Project, the introduction of a hierarchical cognitive cycle to enable multi-tier self-management in various NEs and dynamic network compartments provides a quite promising approach to alleviate management overhead, ensure dynamic adaptation to service requirements, situation aware NM and reconfiguration, while coping with the fragmentation of contemporary centralised NM, dedicated to specific types of networks ([26], [27]). NM is a wide area, including device monitoring, service levels and application management, security, ongoing maintenance, troubleshooting, planning, and other tasks - ideally all coordinated and supervised by an experienced and reliable "entity" (known as the "network administrator"). For businesses of all sizes, it is imperative to consider a NM solution that is easy to use, quick to deploy, and offers low total cost of ownership. Adoption of appropriate cognitive techniques on different platforms (or on parts of them) can be the "kick-off" that will encourage the creation of new networking infrastructures. Furthermore, it is essential to perform NM activities in a distributed way by incorporating self-organization and self-management principles [28]. Although there is a diversity of external and influencing available definition on self-management related work [29], the term "self-management" is applied here as "the general term describing all autonomic and cognition-based operations in a system". Six distinct methods are identified with specific realizations and purposes; they all serve to demonstrate concepts inherent in the system properties ([19], [22]). 


\section{Challenges and Benefits for the Market Sector}

The implementation-inclusion of suitable cognitive techniques/systems on diverse platforms can be the "first step" to support development of new networking infrastructures [30]. The introduced -by the Self-NET-functionalities can implicate major benefits for all relevant "actors" (i.e. for both operators and users), as follows:

Automatic network planning and reduction of management time of complex network parameters (and/or structures): Both present and future anticipated high proliferation of different services that a communications network should offer and support; this imposes a decisive challenge for any network operator involved, while implicating an appropriate "adjustment" of network performance together with "optimization" of the network resources usage. Daily (human) network manager activities include many tedious and time-consuming tasks, to make certain that the network delivers the desired services to its users. In many cases, the network operator is obliged to search through vast amounts of monitoring data to find any "inconveniences" to his network behaviour and to ensure a proper services' delivery. Embedding selfmanagement functionalities in future NEs and establishing cognition at the diverse network levels (e.g., NEs, network compartments and domains) can automate the detection of any abnormal (or "adverse") behaviour, the remoteness of the relevant source(s), the diagnosis of the corresponding fault(s) and the expected repair of the conceived problematic situation. For a variety of reasons affecting the competitive presence of an operator in the market sector, it is a matter of high importance for the network to be able to "predict" irregular events (like faults or intrusions) and so to react, accordingly, in due time. Thus, applying self-aware techniques in a modern network environment can ease network composition and network planning procedures and can ensure the automatic adaptation of networks/services to capabilities of the network components.

Options for reduction of network operational cost: Any infrastructure that can perform automated operational tasks to optimize its network efficiency and the quality of service(s) offered, can contribute to the objective of reducing actual network operational expenditures (OPEX). The option for automating several procedures can be remarkably beneficial to network operators as it facilitates various complex (and resource-consuming) processes, currently deployed at a large time-scale and requiring significant human intervention. This also allows for a more inexpensive and simpler network deployment: That is, by applying self-management techniques intending to optimize the network in terms of coverage, capacity, performance etc., operators can decrease their operational expenditures by limiting the manual effort required for network operation and can actively utilize their NEs (or resources) more efficiently. Such techniques can also simplify network maintenance and fault management.

Options for easy "network adaptation" (e.g., in new traffic models and schemes): Traffic management of a communications network is mainly based on integrated and centrally coordinated deployment of specific measures and suitable rules, in response to the current network operating state and/or in anticipation of future needs and relevant conditions. Traffic management configuration of large wireless networks consisting of multiple, distributed NEs of varying technologies, is challenging, time- 
consuming, prone to possible errors and requires highly expensive control \& management equipment from any market actor. Even when it is originally deployed, it involves continuous upgrading/modifications to provide a consistent and a transparent service environment, to sustain high QoS, to recover from faults and to maximize the overall network performance, especially when congestion phenomena appear.

Seamless users' experience in dynamic network selection: In competitive markets, end-users wish to have access to a network offering adequate coverage and services of high quality, on a real-time basis. Self-management can offer decentralized monitoring and proper decision-making techniques so that appropriate optimization hints can be extracted, in terms of determining the optimum course of actions to improve network performance and stability and to guarantee service continuity.

Enhanced service provision \& adaptability: Dynamic detection of operational deficiencies and/or poor QoS delivered to the end-user, both imply for specific remediate actions to compensate for the related problematic situation(s). Improving the overall network quality also increases subscribers' satisfaction \& trust. Thus, the optimization of all procedures in order to "minimize" (or occasionally to "delete") service failures and to ensure continuity of service delivery is a critical matter for the user and the operator, in a competitive market. Besides, it is quite important for the entire network to incorporate options and other NM facilities to fulfil any requirement for novel service features, such as network (or service) reconfiguration capabilities, broadband management and support of an increased set of services/facilities offered.

Enabling effective networking under highly demanding conditions: A continuous and dynamically updated NM (proactively and reactively adapted to the network dynamics) is an appropriate tool for such purpose. That is, instead of using manual techniques, a fully automated, transparent and intelligent traffic management functionality can be much more beneficial. The Self-NET infrastructure can be used to provide an efficient real-time traffic management in a large network, thus maximizing network performance and radically decreasing human intervention. Several among the application areas can cover cases of traffic congestion, network attachments, link failures, performance degradation, mobility issues, multi-service delivery enhancements and involve intelligent autonomic congestion management and traffic routing, dynamic bandwidth allocation \& dynamic spectrum re-allocation [22].

The continuity of service availability influences directly the technical approach of service realization and is an important parameter affecting network planning; indeed, the network should possess fitting techniques to "adapt itself" to an essential (occasionally prescribed) functional state. To this aim, the network should be able to gather information about various entities (elements, domains, sectors) and/or distinct modules, to detect their operational state(s) and to react to any deviations from the proposed "desired" state. Applying self-aware mechanisms can conduct to network performance optimization in terms of coverage and capacity, optimization of QoS delivered to the end-user and reduction of human intervention [31]. This option can contribute to guarantee some critical features including, but not limited to: (i) High availability \& seamless services' continuity; (ii) Connectivity anywhere and anytime; (iii) Robustness and stability/steadiness of the underlying network; (iv) Scalability in terms of features-functions; (v) Balance between cost network-related benefits (OPEX reduction and optimized network functionalities), and; (vi) Heterogeneity support. 


\section{Experimental Results for Network Coverage and Optimization}

In current practice, wireless network planning is a difficult and challenging task, involving expert knowledge and profound understanding of the factors affecting the performance of a wireless system. Several monitoring parameters should be taken into account for optimal coverage and capacity formation, while diverse configuration actions can be available that in many cases are interrelated, as regards the consequences. In established approaches, frequency planning is conducted as part of the deployment procedure for full network segments (or domains). The assignment of operating frequencies and/or channels to NEs is also a part of the broader frequency planning procedure. To eliminate conflicts in frequency assignment, the process is centrally coordinated in one that assumes and requires global knowledge and control over the concerned network segment(s)/domain(s). In this context, the latter option implies that the administrative entities are fully aware of the channel assigned to each individual NE and are fully capable of "adjusting" such assignments to their liking in a centrally coordinated manner. As a result, conflicts may be avoided or, at least, minimized, when a central entity coordinates and manages the entire procedure.

During the Self-NET Project effort, an extended experimental work has also been performed upon several specific use cases that have all been selected as appropriate "drivers-enablers" for testing and validation activities. A characteristic use case, particularly studied, was relevant to the challenge for achieving "coverage and capacity optimization", for the underlying network. In fact, management systems of modern FI networks incorporate autonomic capabilities to effectively deal with the increasing complexity of communication networks, to reduce human intervention, and to promote localized resource management. The Self-NET framework is based on the Generic Cognitive Cycle, which consists of the "M-D-E" phases. The Network Element Cognitive Manager (NECM) implements the MDE cycle at the NE level, whilst the Network Domain Cognitive Manager (NDCM) manages a set of NECMs, thus implementing sophisticated MDE cycle features. In order to test the key functionalities of the proposed solution, specific NM problems have been taken into account, under the wider scope of wireless networks coverage and capacity optimization family [32]. In the proposed test-bed, a heterogeneous wireless network environment has been deployed, consisting of several IEEE 802.11 Soekris access points (AP) [33] and an IEEE 802.16 Base Station (BS) [34], each embedding a NECM. Moreover, several single radio access terminals-RATs (i.e. Wi-Fi) and multi-RATs (i.e. WiFi, WiMAX) were located in the corresponding area, consuming a video service delivered by VLC (video LAN client) -based service provider [35]. For the management of the NECMs, a NDCM has been deployed. The cognitive network manager installed per NE has undertaken several distinct actions, that is: (i) The deductions about its operational status; (ii) the proactive preparation of solutions to face possible problems, and; (iii) the fast reaction to any problem by enforcing the anticipated reconfiguration actions. Interaction of NECMs and NDCM enabled the localized and distributed orchestration of various NEs. In the experimentation phase we focused on the (re-)assignment of operating frequencies to wireless NEs and the vertical assisted handover of multi- 
RATs. The demonstration scenario has been divided into: (i) The optimal deployment of a new WiFi AP; (ii) the self-optimization of the network topology through the assisted vertical handover of terminals from loaded to neighbouring -less loaded- APs or BS(s), and; (iii) the self-optimization of the network topology due to high interference situation. The MDE cycle was instantiated in both the NECM and the NDCM. The NECM periodically monitored its internal state and local environment by measuring specific parameters, thus building its local view. All NECMs have periodically transmitted the collected information to the NDCM in order to enable, the latter, to "build" the second level of situation awareness (SA) and have the domain level view. The topology used and the allocation of network devices in a realistic office environment (at OTE's R\&D premises), have both been considered as shown in Fig.3. The topology has been selected in order to be "characteristic" and to depict conditions that are common to corporate environments and, especially to those that can occasionally host numerous nomadic end-users.

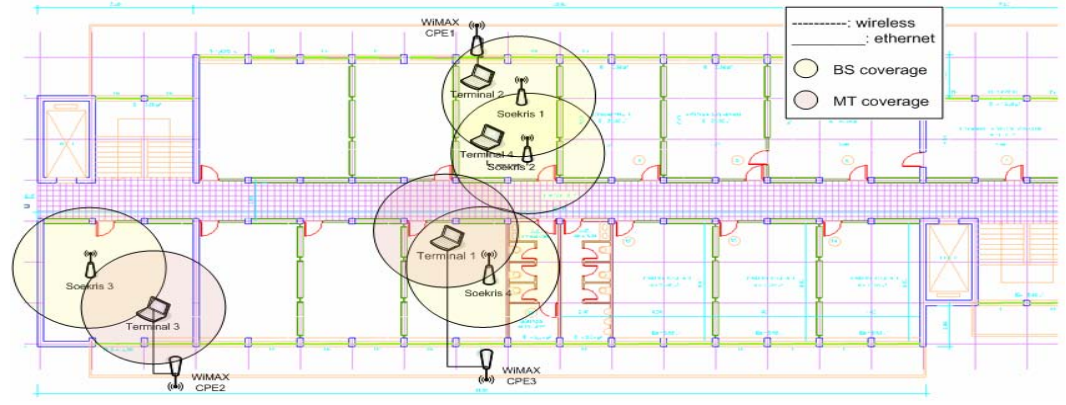

Fig. 3. Network Topology of the proposed Use Case for Coverage and Capacity Optimization.

Fig. 4 illustrates the total duration of the channel selection that takes place with the activation of an AP. It is shown that Soekris 1 and Soekris 4 need more time for channel selection. The most time consuming processes are Execution and Communication. The communication phase is responsible for Soekris 1 and Soekris 4 high delay. Specifically, both Soekris interact (i.e. communicate) with Soekris 2. The duration of Execution phase is high and the same for all devices due to technical and implementation reasons. Moreover, the "decision-making” phase (i.e. Channel Selection Objective Function) is too low for all NECMs, while the monitoring phase takes between 2.55-3.15 seconds. The communication between NECMs increases the duration of the communication phase, while the duration of the execution phase is increased, due to technical and implementation reasons.

Fig. 5 presents the duration of the mobile terminal re-allocation function (vertical assisted handover). This problem solving process is selected if a high load status has been identified. Similarly to the previous cases (i.e. the channel (re-)selection) the communication phase takes again the majority of time.

The proposed test-bed has demonstrated that the inclusion of the MDE cognitive cycle can provide several major operational benefits, such as: (i) Automated installation of wireless access devices with avoidance of overlapping among them; this is 


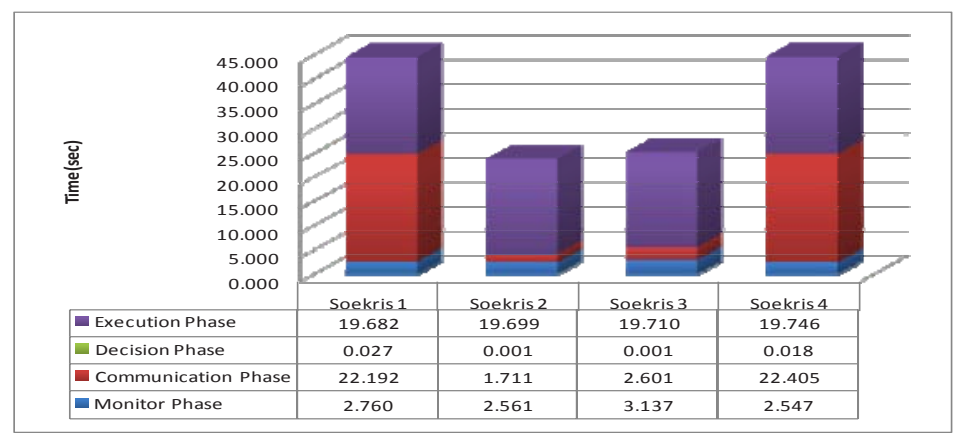

Fig. 4. Channel Selection Duration.

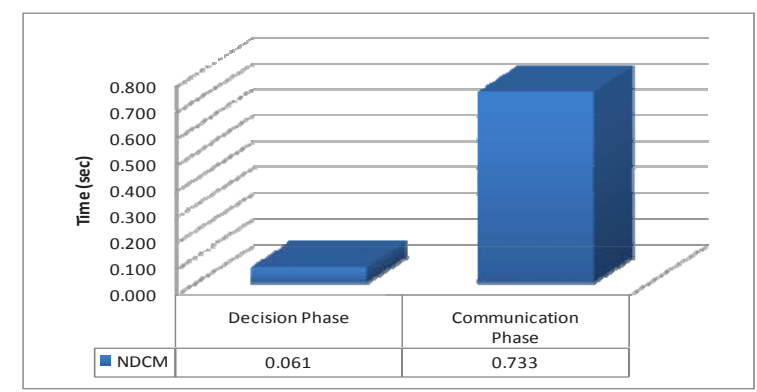

Fig. 4. Vertical Assisted Handover Duration.

done without any human intervention for channel selection. (ii) Automated channel (re-)selection which is made after consideration/evaluation of the existing interference in a specific location of the network. (iii) Automated optimization process for channel (re-)selection, according to specific parameters (i.e.: the number of end-users, the network traffic, the operational state of neighboring access devices, etc.); this can guarantee a more improved functionality for all network devices involved. (iv) Possibility for automated handover between end-users of heterogeneous wireless technologies (WiFi, WiMAX), especially in cases where there is "extreme" network traffic and/or overload, affecting the network functionality.

\section{Conclusion}

Evolution towards FI requests a more flexible architecture that will act as the "basis" for the disposal of a multiplicity of services-facilities with optimized quality levels, intending to attract/satisfy end-users. Such network infrastructures are characterized by the inclusion of embedded intelligence "per element" or "per domain", targeting at a more distributed environment both in terms of management and operational activities. To this aim, cognitive networks with self-aware functionalities introduce a high level of autonomy, meaning that embedded and/or inherent management functionality in several components of FI systems composes management upon a "per 
$N E$ " and/or a "per domain" mechanism, rather than a centralized (traditional) network functionality. Compared to current network features, self-management techniques pave the way towards automated network processes such as the deployment of new NEs, the network reconfiguration (in whole or in part) and the selection/execution of the optimal corresponding solution (or "response") based on specific circumstances \& remediation of identified malfunctions with the minimum potential service interruption. Consequently, new methods (related to embedded and/or autonomous management, virtualization of systems and network resources, advanced and cognitive networking of information objects), have to "re-define" the overall FI network architecture. To "encounter" such critical challenges, the main objective of Self-NET project effort is to describe and evaluate/analyze new paradigms for the management of complex and heterogeneous network infrastructures-systems (such as cellular, wireless, fixed and IP networks), taking into consideration the next generation Internet environment and the convergence perspective. This can efficiently integrate new operational capabilities in the "underlying system" by introducing innovative selfmanagement attributes, resulting in noteworthy benefits for all actors involved. The Self-NET initiative develops self-management features that alleviate consequences of events for which the system would require various invocations of remedy actions and/or significant human intervention. This dynamic behavior and intelligence of handling various events (and/or situations) can potentially lead to an innovative and much promising beneficiary scope of the entire system's operations.

Acknowledgments. The present work has been composed $\mathrm{n}$ the context of the SelfNET ("Self-Management of Cognitive Future Internet Elements") European Research Project and has been supported by the Commission of the European Communities, in the scope of the $7^{\text {th }}$ Framework Programme ICT-2008, Grant Agreement No.224344.

Open Access. This article is distributed under the terms of the Creative Commons Attribution Noncommercial License which permits any noncommercial use, distribution, and reproduction in any medium, provided the original author(s) and source are credited.

\section{References}

1. Commission of the European Communities: Communication on "A Public-Private Partnership on the Future Internet". European Commission, Brussels (2009)

2. Chochliouros, I.P., Spiliopoulou, A.S.: Broadband Access in the European Union: An Enabler for Technical progress, Business Renewal and Social Development. The International Journal of Infonomics (IJI) 1, 5-21 (2005)

3. Timmers, P.: Business Models for Electronic Markets. The International Journal on Electronic Markets and Business Media 8(2), 3-8 (1998)

4. Future Internet Assembly (FIA): Position Paper: Real World Internet (2009), http://rwi.future-internet.eu/index.php/Position_Paper

5. Afuah, A., Tucci, C.L.: Internet Business Models and Strategies: Text and Cases. McGraw-Hill, New York (2000)

6. European Future Internet Portal (2010), http: / /www. future-internet.eu/ 
7. Blumenthal, M.S., Clark, D.D.: Rethinking the Design of the Internet: The End-to-End Arguments vs. the Brave New World. ACM Trans. on Internet Techn. 1(1), 70-109 (2001)

8. Commission of the European Communities: Communication on "The Future EU 2020 Strategy". European Commission, Brussels (2009)

9. Tselentis, G., Domingue, L., Galis, A., Gavras, A., et al.: Towards the Future Internet-A European Research Perspective. IOS Press, Amsterdam (2009)

10. Organization for Economic Co-operation Development (OECD): The Seoul Declaration for the Future of the Internet Economy. OECD, Paris, France (2008)

11. Chochliouros, I.P., Spiliopoulou, A.S.: Innovative Horizons for Europe: The New European Telecom Framework for the Development of Modern Electronic Networks and Services. The Journal of the Communications Network (TCN) 2(4), 53-62 (2003)

12. Commission of the European Communities: Communication on "Future Networks and the Internet". European Commission, Brussels (2008)

13. Galis, A., Brunner, M., Abramowitz, H.: MANA Position Paper - Management and Service-Aware Networking Architecture (MANA) for Future Internet / Draft 5.0 (2008)

14. International Telecommunication Union-Telecommunication Standardization Sector: Rec. M. 3400: TMN Management Functions. ITU-T, Geneva, Switzerland (2000)

15. Pastor-Satorras, R., Vespignani, A.: Evolution and Structure of the Internet: A Statistical Physics Approach. Cambridge University Press, Cambridge (2004)

16. Dobson, S., Denazis, S., Fernandez, A., et al.: A survey of autonomic communications. ACM Trans. on Autonomous and Adaptive Systems (TAAS) 1(2), 223-259 (2006)

17. Boccalettia, S., Latora, V., Moreno, Y., Chavez, M., Hwang, D.-U.: Complex networks: Structure and Dynamics. Elsevier Physics Reports 424, 175-308 (2006)

18. Clark, D., Sollins, K., Wroclawski, J., Katabi, D., et al.: New Arch: Future Generation Internet Architecture (Final Technical Report). The US Air Force Research Laboratory (2003)

19. Chochliouros, I.P., Spiliopoulou, A.S., Georgiadou, E., Belesioti, M., et al.: A Model for Autonomic Network Management in the Scope of the Future Internet. In: Proceedings of the 48th FITCE International Congress, FITCE, Prague, Czech Republic, pp. 102-106 (2009)

20. Kousaridas, A., Polychronopoulos, C., Alonistioti, N., et al.: Future Internet Elements: Cognition and Self-Management Design Issues. In: Proceedings of the 2nd International Conference on Autonomic Computing and Communication Systems, pp. 1-6 (2008)

21. Raptis, T., Polychronopoulos, C., et al.: Technological Enablers of Cognition in SelfManageable Future Internet Elements. In: Proceedings of The First International Conference on Advanced Cognitive Technologies and Applications COGNITIVE 2009, pp. 499 504. IARIA (2009)

22. Mihailovic, A., Chochliouros, I.P., Kousaridas, A., Nguengang, G., et al.: Architectural Principles for Synergy of Self-Management and Future internet Evolutions. In: Proceedings of the ICT Mobile Summit 2009, pp. 1-8. IMC Ltd, Dublin (2009)

23. Self-NET Project: Deliverable D1.1: System Deployment Scenarios and Use Cases for Cognitive Management of Future Internet Elements (2008), https : / www . ict-selfnet. eu/

24. Agoulmine, N., Balasubramaniam, S., Botvitch, D., Strassner, J., et al.: Challenges for Autonomic Network Management. In: Proceedings of the 1st IEEE International Workshop on Modelling Autonomic Communications Environments (2006)

25. Strassner, J.: Policy-Based Network Management. Morgan Kaufmann Publishers, San Francisco (2003)

26. Elliott, C., Heile, B.: Self-organizing, self-healing wireless networks. In: Proceedings of IEEE International Conference on Personal Wireless Communications, pp. 355-362 (2000) 
27. Chochliouros, I.P., Alonistioti, N., Spiliopoulou, A.S., et al.: Self-Management in Future Internet Wireless Networks: Dynamic Resource Allocation and Traffic Routing for MultiService Provisioning. In: Proceedings of MOBILIGHT-2009, pp. 1-12. ICST (2009)

28. Self-NET Project: Deliverable D5.1: First Report on Business Opportunities (2009)

29. Miller, B.: The autonomic computing edge: Can you CHOP up autonomic computing? IBM Corporation (2008)

30. Prehofer, C., Bettstetter, C.: Self-Organization in Communication Networks: Principles and Design Paradigms. IEEE Communications Magazine 43(7), 78-85 (2005)

31. Mihailovic, A., Chochliouros, I.P., Georgiadou, E., Spiliopoulou, A.S., et al.: Situation Aware Mechanisms for Cognitive Networks. In: Proceedings of the International Conference on Ultra Modern Telecommunications (ICUMT-2009), pp. 1-6. IEEE Computer Society Press, Los Alamitos (2009)

32. Pragad, A.D., Friderikos, V., Pangalos, P., Aghvami, A.H.: The Impact of Mobility Agent based Micro-Mobility on the Capacity of Wireless Access Networks. In: Proceedings of IEEE GLOBECOM-2007 (2007)

33. Soekris Engineering net5501, http://www. soekris.com/net5501.htm

34. RedMAX, Redline Communications: AN-100U/UX Single Sector Wireless Access Base Station User Manual (2008)

35. C.: open-source multimedia framework, player and server, http://www. videolan.org/vlc 